\title{
DETERMINACIÓN DE CONTENIDO DE HUMEDAD EN ASTILLAS DE PINUS CARIBAEA VAR. HONDURENSIS, UTILIZANDO UN HORNO DOMÉSTICO DE MICROONDAS

\author{
Pedro R. Benítez Díaz
} Ilvania Gutiérrez Gotera
}

\begin{abstract}
Resumen
Es necesario que la industria papelera se encamine por el sendero de la química e ingeniería verde, para ello se requiere desarrollar métodos de análisis más eficientes que consuman menos energía y sean amigables con el ambiente. En este trabajo se desarrolló un método para la determinación del contenido de humedad en astillas de Pinus caribaea var. hondurensis, utilizando una fuente de energía alternativa como es la radiación de microondas, empleando para ello un horno de microondas doméstico con una potencia de cocción de 1.200 W. Con el método desarrollado se logró un tiempo total de análisis de aproximadamente dos horas, la fuente de energía se utilizó sólo doce minutos y el consumo eléctrico fue de $0,27 \mathrm{kWh}$ por cada análisis, con respecto al método convencional se redujo el tiempo de análisis en $90 \%$ y el consumo de energía eléctrica en 99\%. Adicionalmente, la aplicación de radiación de microondas mostró ser de 16\% a 34\% más eficiente que la estufa en la extracción de la humedad de las astillas. El desarrollo de este tipo de metodologías incidirá positivamente en la optimización de los procesos y control de calidad de la industria papelera.
\end{abstract}

Palabras clave: humedad de astillas, microondas, sostenibilidad, pulpa y papel, química verde.

\begin{abstract}
Due to the need of the paper industry to follow the path of green chemistry and engineering, it is necessary to develop more efficient analytical methods that consume less energy and are more environmentally friendly than traditional methods. In this paper, we developed a method for determining the moisture content in wood chips of Pinus caribaea var. hondurensis using an alternative energy source, such as microwave radiation, employing a domestic microwave oven with a cooking power of $1,200 \mathrm{~W}$. With the developed method, we achieved a total analysis time of approximately two hours, the energy source was used for only twelve minutes and the power consumption was 0.27 $\mathrm{kWh}$ per analysis; with respect to the conventional method, the time of analysis was reduced by $90 \%$ and electricity consumption, by $99 \%$. Additionally, the application of microwave radiation was shown to be $16 \%$ to $34 \%$ more efficient than the furnace in the extraction of moisture from the wood chips. The development of such methods has a positive impact on the optimization of processes and quality control of the paper industry.
\end{abstract}

Keywords: woods chips moisture, microwave, sustainability, pulp and paper, green chemistry.

Recibido: 14 de Febrero del 2012 • Aprobado: 15 de Mayo del 2012

\section{INTRODUCCIÓN}

La industria papelera, al igual que muchas otras industrias que requieren de la utilización de productos químicos peligrosos, genera emisiones y efluentes contaminantes a la atmósfera, ríos y mares (Gaete, Bay-Schmith \& Riveros, 2005; Ferluc, 2008). Adicionalmente, este tipo de industria requiere de grandes cantidades de energía, sobre todo en las áreas de pulpificación, blanqueo, refinación y secado; consumiendo una gran cantidad de recursos energéticos no renovables (Area, 2005; Soto, 2005). La respuesta de la industria de pulpa y papel a la demanda social sobre el cuidado del ambiente no se ha hecho esperar y en la última década se ha trabajado en la modificación y desarrollo de procesos amigables con el ambiente y ahorradores de energía (Area, 2005; Battana, Sharma, Sudha \& Chander, 2007; Cerón, 2009). 
Esta respuesta se debe en parte a una tendencia que, desde los años noventa, viene cambiando la forma de abordar el problema ambiental. Actualmente, en lugar de remediar la contaminación ya producida y controlar emisiones y efluentes pensamos que es mejor prevenir, en otras palabras, es preferible trabajar e invertir para evitar las emisiones, el despilfarro de energía y el manejo de productos químicos indeseables en lugar de reparar las consecuencias de conductas inadecuadas (Meléndes \& Camacho, 2008). Esta actitud originó lo que hoy conocemos como química e ingeniería verde, también llamadas química e ingeniería sostenibles, cuyo objetivo es promover el desarrollo y la utilización de tecnologías y metodologías innovadoras que permitan un empleo más eficiente de la energía y reduzcan o eliminen la utilización o generación de sustancias dañinas para el ambiente, bien sea en el diseño o manufactura de cualquier producto de consumo o en los procesos químicos (American Chemical Society, 2001).

Como consecuencia de este movimiento, se han empezado a desarrollar tecnologías para optimizar la utilización de la energía en los procesos químicos, esto se ha logrado empleando fuentes alternativas de energía como la radiación de microondas. La utilización de microondas como fuente de energía térmica, puede incrementar la eficiencia y reducir el tiempo de muchos procesos (Breccia, Fini, González \& González, 2003; Bélanger et al., 2008; Kappe y Dallinger, 2009). La penetración de las microondas permite un calentamiento interno y rápido, reduciendo las pérdidas de energía que ocurren en los procesos de transferencia de calor que comúnmente conocemos, como es el caso de las estufas y mantas de calentamiento, entre otros (Ayappa, Davis, Davis \& Gordon; 1991). Más recientemente, han aparecido artículos científicos que proponen diferentes tipos de aplicaciones de las microondas en la industria forestal. Estas aplicaciones incluyen modificaciones químicas de la madera (Rowell, 2007),preservación (Schmidt, 2006; Treu, Rieche \& Militz, 2008), secado y determinación de humedad de la madera en forma más eficiente (James, Yen \& Kin, 1985; Du, Wang \& Cai, 2005; Hunt, Gu, Walsh \& Winandy, 2005).

En este contexto, en el Laboratorio de Química del Laboratorio Nacional de Productos
Forestales (LNPF) de la Universidad de Los Andes, Mérida-RB de Venezuela, se ha tratado de desarrollar una metodología verde para la determinación del factor seco o el contenido de humedad en astillas que serán utilizadas en la fabricación de pulpa para papel. Conocer la humedad en astillas es importante para calcular los costos de producción, el control de inventarios de materia prima y la determinación tanto de la carga de astillas como de álcali que será utilizado en el proceso (TAPPI, 1998). Tradicionalmente, el procedimiento utilizado requiere del empleo de estufas que deben permanecer encendidas por lo menos 20 horas a una temperatura promedio de $105^{\circ} \mathrm{C}$, este método está desarrollado a partir de las normas T 258 om-94 y T 264 cm-97 (TAPPI, 1998), y ASTM D-4442-92 (ASTM D-444292, 2003). Es común en los laboratorios de las industrias que las estufas permanezcan encendidas 24 horas siete días de la semana, con la finalidad de tener los equipos listos para realizar los análisis cuando sea requerido. Las estufas utilizadas, pueden tener un consumo de energía que varía entre 820 y $1.200 \mathrm{~W}$, lo que supone un consumo diario de energía que varía entre 19,98 y $28,8 \mathrm{kWh}$.

El presente trabajo, tiene como objetivo desarrollar un método para la determinación del factor seco o contenido de humedad de astillas de Pinus caribaea var. hondurensis, empleando como fuente de energía térmica la radiación del microondas.

\section{MÉTODOS}

\section{Equipos}

Balanza analítica con apreciación de 0,1 mg marca OHAUS Explorer. Estufa con control de temperatura marca K Gemmy Industrial. Horno de microondas doméstico marca Panasonic Inverter, modelo NNST678 S, potencia de la fuente de poder: $1.480 \mathrm{~W}$, potencia de cocción $1.200 \mathrm{~W}$, frecuencia: 2.450 MHz, capacidad: 33 L (225 mm X 375 mm X $386 \mathrm{~mm}$ ). Termómetro infrarrojo digital marca Fluke Modelo 568. Pesafiltros de $60 \mathrm{ml}$ con tapa esmerilada. 


\section{Muestras}

La fuente de las muestras fueron dos árboles de Pinus caribaea var. hondurensis de 35 años de edad, que se talaron de los alrededores del LNPF, previo trámite del permiso correspondiente a través del Ministerio del Poder Popular para el Ambiente. Estos árboles fueron cortados en trozas de $2,5 \mathrm{~m}$, las cuales fueron numeradas aleatoriamente. Para evitar la pérdida de humedad, se mantuvo la corteza en las trozas y sus extremos se sellaron con pintura acrílica. Posteriormente, se escogieron las trozas 10 y 11 (R10 y R11), para aserrarlas con la finalidad de obtener cuartones de $8 \mathrm{~cm} \times 8 \mathrm{~cm}$ x $60 \mathrm{~cm}$, los cuales fueron numerados aleatoriamente $\mathrm{y}$ astillados en una astilladora Carthage. Las características de las astillas obtenidas fueron las siguientes: longitud promedio $21 \mathrm{~mm}$ con un coeficiente de variación (CV) de $12 \%$, ancho promedio $29 \mathrm{~mm}$ con un CV de $24 \%$ y espesor promedio de $3,8 \mathrm{~mm}$ con un CV de $18 \%$. Las astillas fueron almacenadas en bolsas plásticas cerradas con la finalidad de mantenerlas saturadas de humedad hasta el momento del análisis.

Para hacer los ensayos de factor seco se escogieron tres muestras al azar: de la troza R10 se tomaron los cuartones 1 y 10 , que identificamos como muestras R10 C1 y R10 C10; y del R11 se escogió el cuartón C3, que identificamos como muestras R11 C3. Debemos resaltar que las astillas se obtuvieron de madera verde y en condiciones de saturación de humedad.

Cuando se seleccionaron las muestras para realizar los ensayos se evitó escoger astillas provenientes de nudos, con crecimiento de hongos y se tuvo particular cuidado de no utilizar muestras contaminadas con restos de metales.

\section{Método convencional para determinación de factor seco (MC)}

Este método fue desarrollado en la Sección de Pulpa y Papel del LNPF, basándose en los métodos de la norma TAPPI y ASTM (TAPPI, 1998; ASTM D-4442-92, 2003). Brevemente: los pesafiltros limpios se colocaron en una estufa a $105 \pm 3^{\circ} \mathrm{C}$, por un período de dos horas, para luego dejarlos enfriar durante $15 \mathrm{~min}$ en un desecador y posteriormente pesarlos en la balanza analítica, este procedimiento se repitió hasta llegar a un peso constante, este peso se reportó como pesafiltro seco (PFS). Paso seguido, los pesafiltros se dejaron al ambiente durante 15 min y se pesaron nuevamente, este peso se reportó como pesafiltro húmedo (PFH). Luego, se introdujeron las astillas en el pesafiltro hasta alcanzar aproximadamente $2 / 3$ de su altura y rápidamente se pesó el conjunto, este peso se identificó como pesafiltro húmedo más muestra húmeda $(\mathrm{PFH}+\mathrm{MH})$. Las muestras se colocaron en la estufa a $105 \pm 3{ }^{\circ} \mathrm{C}$ por un período no menor a 16 horas, posteriormente se dejaron enfriar en el desecador durante 15 min y se pesaron, el procedimiento se repitió con intervalos de calentamiento de una hora hasta obtener peso constante (se aceptó sólo variación en la tercera cifra decimal), este peso se identificó como pesafiltro seco más muestra seca (PFS + MS). Se hicieron determinaciones por triplicado en paralelo para cada muestra y se realizaron los cálculos correspondientes según las ecuaciones que se muestran a continuación:

$\mathrm{FS}=\frac{(\mathrm{PFS}+\mathrm{MS})-\mathrm{PFS}}{(\mathrm{PFH}+\mathrm{MH})-\mathrm{PFH}}$

donde:

$\mathrm{FS}=$ Factor de sequedad

PFS $=$ Pesafiltro seco

MS = Muestra seca

$\mathrm{PFH}=$ Pesafiltro húmedo

$\mathrm{MH}=$ Muestra húmeda

$\% \mathrm{HP}=(1-\mathrm{FS}) * 100$

donde:

$\%$ HP = Porcentaje de humedad perdida en base al peso húmedo

FS $=$ Factor de sequedad

Para determinar factor de sequedad utilizando un horno de microonda se desarrollaron dos métodos el HMO-1 y HMO-2, los cuales se describen a continuación.

\section{Método HMO-1}

Con este método se determinó el factor de sequedad por quintuplicado, colocando una astilla en cada pesafiltro y utilizando el 
horno de microondas en su máxima potencia $(1.200 \mathrm{~W})$. El tiempo de exposición a la radiación electromagnética, se controló a través del cronómetro incorporado en el horno de microondas. Para la determinación del peso seco de los pesafiltros, se colocaron cinco pesafiltros limpios y debidamente rotulados dentro del horno de microondas y se sometieron a calentamiento según el procedimiento mostrado en el Cuadro 1. El paso tres se repitió hasta obtener peso constante (se aceptó sólo variación en la tercera cifra decimal), se anotó este peso como pesafiltro seco. Posteriormente, los pesafiltro se dejaron reposar al aire sobre una superficie libre de polvo, por un período de 15 minutos y se volvieron a pesar en la balanza analítica, anotando este peso como pesafiltro húmedo.

Seguidamente, se seleccionaron cinco astillas saturadas de humedad cuyos pesos variaron entre 1,2 y $1,5 \mathrm{~g}$. Se colocó cada astilla en un pesafiltro y se pesaron nuevamente en la balanza analítica anotando este peso como pesafiltro húmedo más muestra húmeda. Luego, las muestras fueron sometidas a períodos de calentamiento (en el horno de microondas) y enfriamiento (desecador) según el procedimiento mostrado en el Cuadro 2 y se repitió el paso 10 hasta que se obtuvo peso constante en todas las muestras, este peso se anotó como pesa filtro seco más muestra seca. El factor de sequedad y el porcentaje de humedad perdida en base al peso húmedo se calcularon utilizando las ecuaciones uno y dos, de igual manera que en el método convencional, tomando la condición de saturación de agua como $100 \%$ de humedad. El tiempo acumulado de exposición a la radiación de microondas se obtuvo sumando sólo los intervalos de tiempo en los cuales las astillas estuvieron sometidas a calentamiento en el horno de microondas. Se midió la temperatura de las astillas inmediatamente después de finalizar cada período de calentamiento utilizando para ello un termómetro infrarrojo.

Es importante destacar que se tuvo el cuidado de mantener la puerta del horno de microondas abierta durante los períodos de enfriamiento, esto permitió desalojar el exceso de vapor de agua que quedaba dentro del mismo después de cada período de calentamiento. También, los períodos de enfriamiento de los dos primeros pasos del procedimiento (Cuadro 2), se realizaron fuera del desecador para evitar la pérdida de actividad del desecante (en nuestro caso sílica gel). Adicionalmente, se tomó la previsión de reactivar el desecante después de cada experimento, lo cual también se hizo empleando el horno de microondas según el siguiente procedimiento: se trasvasó la sílica gel a un vaso de precipitado de $600 \mathrm{ml}$ y se sometió a calentamiento durante dos períodos seguidos de 3 minutos a máxima potencia.

\section{Método HMO-2}

Con este método se determinó el factor de sequedad por triplicado colocando varias astillas en cada pesafiltro y al igual que el caso anterior, se utilizó el horno de microondas a su

Cuadro 1. Procedimiento de calentamiento y enfriamiento en el desecador que se utilizó para obtener el peso seco de los pesafiltros utilizando el horno de microondas (HMO, 1.200 W)

\begin{tabular}{ccc}
\hline Paso & Tiempo en el HMO & Tiempo en el desecador \\
1 & $2 \mathrm{~min}$ & 5 min \\
2 & $1 \mathrm{~min}$ & 15 min y pesar \\
\hline
\end{tabular}


máxima potencia de cocción $(1.200 \mathrm{~W})$. Los valores de pesafiltro seco y pesafiltro húmedo se determinaron con el mismo procedimiento utilizado en el método HMO-1 (Cuadro 1).

Posteriormente, se introdujeron en cada pesafiltro la cantidad de astillas, saturadas de humedad, necesarias para obtener un peso de muestra no menor a $4,3 \mathrm{~g}$ y no mayor a $4,5 \mathrm{~g}$. Se pesó nuevamente en la balanza analítica y se anotó este peso como $\mathrm{PFH}+\mathrm{MH}$. Las muestras fueron sometidas a calentamiento en el horno de microondas y posterior enfriamiento, según el mismo procedimiento empleado en el método HMO-1 (Cuadro 2), hasta que se obtuvo el peso constante en todas las muestras, este peso se anotó como PFS + MS. El FS y $\%$ HP se calcularon utilizando las ecuaciones 1 y 2 , de igual manera que con MC. Se midió la temperatura de las astillas a la salida de cada período de calentamiento, utilizando para ello un termómetro infrarrojo.

\section{Reporte de resultados}

Los resultados fueron reportados como el promedio del factor seco más o menos la desviación estándar (DS). Las diferencias estadísticamente significativas se determinaron a través de un análisis de varianza (ANOVA de una vía) empleando el programa GraphPadInStat.

\section{RESULTADOS}

\section{Calentamiento por exposición a microondas}

Al utilizar un horno de microondas como fuente de energía térmica para la determinación del factor de sequedad, se observó que cuando el tiempo de exposición a la radiación es mayor a un minuto, las astillas empiezan a perder resina y probablemente extraíbles. Cuando este tiempo

Cuadro 2. Procedimiento de calentamiento y enfriamiento en el desecador que se utilizó para determinar el peso seco de las astillas utilizando horno de microondas (HMO, 1.200 W)

\begin{tabular}{ccc}
\hline Paso & Tiempo en el HMO & Enfriamiento \\
\hline 1 & $1 \mathrm{~min}$ & 5 min al aire \\
2 & $1 \mathrm{~min}$ & 5 min al aire \\
3 & $1 \mathrm{~min}$ & 5 min en desecador \\
4 & $30 \mathrm{~s}$ & 5 min en desecador \\
5 & $30 \mathrm{~s}$ & 5 min en desecador \\
6 & $30 \mathrm{~s}$ & 5 min en desecador \\
7 & $30 \mathrm{~s}$ & 5 min en desecador \\
8 & $30 \mathrm{~s}$ & 5 min en desecador \\
9 & $30 \mathrm{~s}$ & 15 min en desecador y pesar \\
10 & $30 \mathrm{~s}$ & 15 min en desecador y pesar \\
\hline
\end{tabular}


excede los dos minutos continuos, la temperatura de las astilla puede alcanzar $130^{\circ} \mathrm{C}$ y si su humedad es baja, pueden llegar a quemarse. Al utilizar el procedimiento de calentamiento descrito en el Cuadro 2, la temperatura de las astillas nunca sobrepasó los $80^{\circ} \mathrm{C}$. En la metodología, también se indicó que la masa total de las astillas que se colocó en cada pesafiltro debía permanecer dentro de un rango, esto se debe a que encontramos que si la diferencia de peso entre ellas es mayor a 0,3 $\mathrm{g}$ algunas muestras llegarán al peso constante más rápido que otras. Adicionalmente, los resultados indican que al utilizar pesafiltros más pequeños o más grandes, el esquema de calentamiento debe ser modificado para obtener buenos resultados en la determinación del factor de sequedad.

\section{Determinación del factor seco}

Los resultados obtenidos para el factor de sequedad a través de los métodos HMO-1 y 2 fueron significativamente menores a los obtenidos por el método convencional (Cuadro 3). Para las muestras $\mathrm{R} 10 \mathrm{C} 1$ y C10, hay una diferencia entre los valores obtenidos por los métodos HMO y el método convencional que en promedio es de 16 $\%$. Para la muestra R11 C3 y C4 esta diferencia es aún mayor, llegando a ser en promedio del $34 \%$. Por otra parte, también encontramos que no hay diferencias estadísticamente significativas entre los resultados del factor de sequedad obtenidos por los métodos HMO-1 y 2, en todas las muestras analizadas. En cuanto a la precisión, los tres métodos tienen coeficientes de variabilidad o deviación estándar relativa por debajo del $5 \%$, lo cual está muy bien para una determinación de humedad donde la variabilidad entre muestras puede llegar a ser muy grande.

En la Figura 1 se muestra el gráfico de porcentaje de humedad perdida en base al peso húmedo en función del tiempo de exposición a la radiación de microondas utilizando el método HMO-2. Se observó que la mayor pérdida de humedad, alrededor del $63 \%$, se registró durante los primeros tres minutos de exposición; entre 3,5 y 5 min la pérdida de humedad es más lenta y varía entre $66 \%$ y $72 \%$; a partir de los 5,5 min las astillas no pierden más humedad y llegan a peso constante.

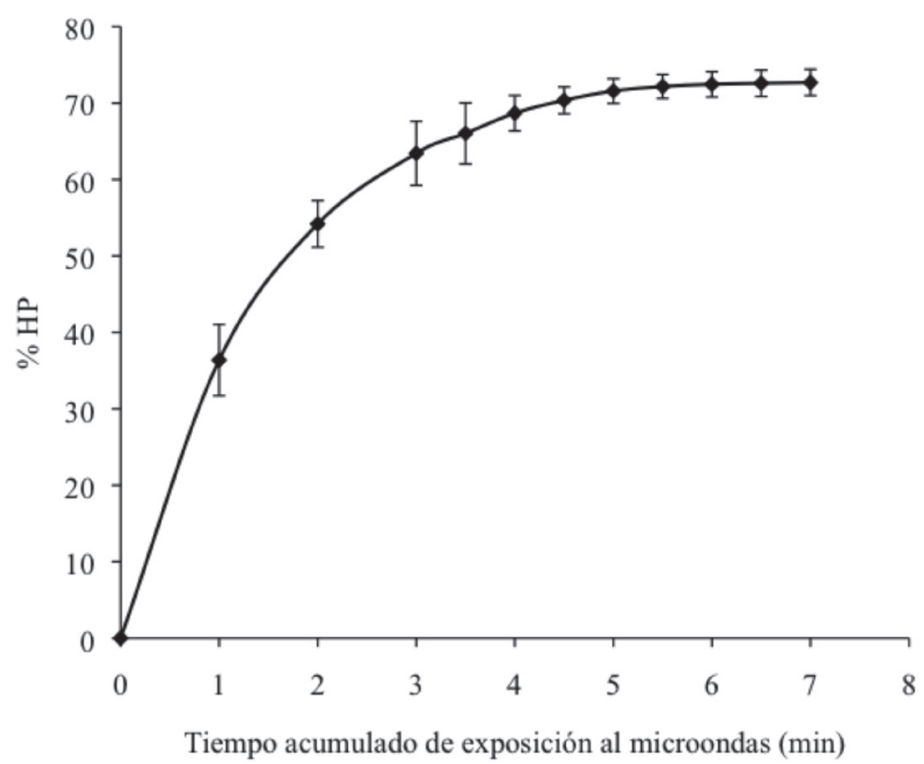

Figura 1. Porcentaje de humedad perdida en base al peso húmedo (\% HP) en astillas de Pinus caribaea var. hondurensis con respecto al tiempo acumulado de exposición a la radiación de microondas. 
Cuadro 3. Comparación de los resultados obtenidos en la determinación del factor de sequedad (FS) y porcentaje de humedad perdida en base al peso húmedo (\% HP) en astillas de Pinus caribaea var. hondurensis utilizando los diferentes métodos estudiados: método convencional (MC), HMO-1 y HMO-2

\begin{tabular}{|c|c|c|c|}
\hline Muestra & Método & $\mathrm{FS} \pm \mathrm{DS}$ & $\% \mathrm{HP} \pm \mathrm{DS}$ \\
\hline & MC & $0,396 \pm 0,009$ & $60,4 \pm 0,9$ \\
\hline \multirow[t]{3}{*}{ R10 C1 } & HMO- $1 * * *$ & $0,327 \pm 0,007$ & $67,4 \pm 0,7$ \\
\hline & HMO- $2 * * *$ & $0,339 \pm 0,012$ & $66,1 \pm 1,2$ \\
\hline & $\mathrm{MC}$ & $0,420 \pm 0,004$ & $58,0 \pm 0,4$ \\
\hline \multirow[t]{3}{*}{ R10 C10 } & HMO- $1 * * *$ & $0,351 \pm 0,018$ & $64,9 \pm 1,8$ \\
\hline & HMO- $2 * * *$ & $0,362 \pm 0,009$ & $63,8 \pm 0,9$ \\
\hline & $\mathrm{MC}$ & $0,454 \pm 0,009$ & $54,6 \pm 0,9$ \\
\hline \multirow[t]{3}{*}{ R11 C3 } & HMO-1*** & $0,309 \pm 0,015$ & $69,1 \pm 1,6$ \\
\hline & HMO- $2 * * *$ & $0,312 \pm 0,009$ & $68,8 \pm 0,9$ \\
\hline & MC & $0,474 \pm 0,010$ & $52,6 \pm 0,9$ \\
\hline \multirow[t]{2}{*}{ R11 C4 } & HMO- $1 * * *$ & $0,316 \pm 0.021$ & $68,2 \pm 3,0$ \\
\hline & HMO-2*** & $0,268 \pm 0,007$ & $73,3 \pm 0,7$ \\
\hline
\end{tabular}

$$
\text { DS = desviación estándar }
$$

*** $\mathrm{P}<0,001$ diferencia estadísticamente significativa cada uno de los métodos HMO y el método convencional. En todos los casos $\mathrm{P}>0,05$ entre HMO-1 y HMO-2, no significativa. Para el método convencional y HMO-1 n = 5, para HMO-2 n = 3.

Fuente: Los autores. 


\begin{abstract}
Ahorro de energía
Empleando cualquiera de los métodos HMO se logró reducir el tiempo de análisis en un $90 \%$, el tiempo de utilización de la fuente de energía térmica en un $99 \%$ y el consumo de energía en un $99 \%$ (Cuadro 4). Aunado a esto, la inversión mínima necesaria para adquirir una estufa a convección forzada es aproximadamente dos mil dólares americanos y la inversión para adquirir un horno de microondas, como el empleado en este trabajo, es de aproximadamente ciento cincuenta dólares americanos (Amazon.com, 2011; ColeParmer, 2011).
\end{abstract}

\section{DISCUSIÓN}

\section{Método de calentamiento por exposición a microondas}

El método de calentamiento por microondas desarrollado en este trabajo para determinar el contenido de humedad de astillas, requiere de la aplicación de una serie de cortos períodos de exposición a la radiación que se alternan con periodos de enfriamiento (Cuadros 1 y 2), en lugar del calentamiento continuo y prolongado en una estufa que requiere el método convencional. Esto se debe a que cuando utilizamos una fuente de energía como la radiación de microondas, el calentamiento ocurre de manera totalmente diferente a lo que ocurre en una estufa (Acierno, Barba \& d'Amore, 2004). En el microondas, el calentamiento se produce por un incremento de la frecuencia de vibración de la molécula de agua, razón por la cual la muestra se calienta prácticamente de forma instantánea y desde el interior (Calvino, Martín \& Lopez, 2008). En estas condiciones se encontró que cuando incrementamos el tiempo de exposición en tan sólo un minuto, puede ocurrir un sobrecalentamiento que trae como consecuencia el deterioro de las muestras y resultados erróneos a la hora del calcular el factor de sequedad. En el procedimiento empleado, el tiempo de reposo que sigue a los cortos periodos de calentamiento, tiene como finalidad que la muestra se enfríe totalmente antes de ser expuesta nuevamente a la radiación de microondas, que es una medida adicional para evitar el sobrecalentamiento. Este procedimiento permite extraer la humedad de las astillas sin elevar la temperatura más allá de $80^{\circ} \mathrm{C}$, evitando la pérdida de compuestos orgánicos volátiles y manteniendo la integridad estructural de la madera, como ha sido destacado por otros autores (Du et al., 2005; Wang, Du \& Zhang, 2005; Hansson \& Antti, 2006).

En un trabajo realizado por $\mathrm{Du} y$ colaboradores, sobre el secado de fibras para fabricar tableros OSB utilizando horno de microondas (Du et al., 2005), quedó claramente establecido que el tiempo de secado y la temperatura que alcanza la muestra durante la

Cuadro 4. Comparación del consumo de energía entre el método convencional (MC) y los métodos por microondas (HMO-1 y 2)

\begin{tabular}{|c|c|c|c|}
\hline Método & Tiempo de análisis & $\begin{array}{c}\text { Tiempo de utilización de la } \\
\text { fuente de energía }\end{array}$ & Consumo de energía \\
\hline $\mathrm{MC}$ & $20 \mathrm{~h}$ & $24 \mathrm{~h}^{*}$ & $19,98-28,80 \mathrm{kWh}^{* *}$ \\
\hline HMO-1 у 2 & $2 \mathrm{~h}$ & $0,18 \mathrm{~h}$ & $0,27 \mathrm{kWh}$ \\
\hline
\end{tabular}

* Generalmente, las estufas se mantienen encendidas las 24 horas al día.

** El consumo nominal de energía de una estufa de laboratorio puede variar entre 820 y $1.200 \mathrm{~W}$. 
exposición a la radiación depende de la relación entre la potencia aplicada y la masa del material que se está secando. Por esta razón, en la metodología se especifica un rango para la masa de astillas que deben ser colocadas en los pesafiltros, si las masas son mayores a las sugeridas se tardará mucho más tiempo en alcanzar el peso constante y si las masas son menores las muestran podrían sobrecalentarse o quemarse. Si la masa de las astillas que se coloca en cada pesafiltro difiere entre sí en más de $0,3 \mathrm{~g}$ algunas muestras llegarán al peso constante más rápido que otras, por tanto el tiempo del análisis no se podrá definir claramente y en el proceso algunas muestras pudieran perder extraíbles o quemarse.

Con respecto a los pesafiltros, aunque estos no participan directamente en el proceso de pérdida de humedad de las astillas, son elementos que también absorben cierta cantidad de energía y se calientan al estar expuestos al microondas, es decir, participan en los fenómenos de intercambio de calor que ocurren durante el proceso; razón por la cual es preferible utilizar los pesafiltros del volumen y características que se indican en la sección de materiales y métodos o ajustar la metodología al tipo de pesafiltro que disponga cada laboratorio.

Se debe dejar claramente establecido que los métodos descritos en este trabajo fueron desarrollados con astillas de Pinus caribaea var. hondurensis que se encontraban saturadas de humedad, para el caso de astillas que se encuentren en condiciones de no saturación, probablemente se haga necesario hacer algunos ajustes en el procedimiento. Esto se debe a que la masa inicial de agua que será calentada al exponer las muestras a la radiación de microondas es mucho menor en la condición de no saturación. Así mismo, probablemente sea necesario hacer ajustes al procedimiento cuando se trabaje con especies diferentes a la empleada para este estudio, debido a las características estructurales y fisicoquímicas particulares de cada una de ellas (Andersson, Persson, Sjoholn \& Svanberg, 2006; Hansson \& Antti, 2006).

\section{Determinación del factor de sequedad}

El hecho de que el factor de sequedad obtenido por los métodos HMO-1 y 2 sea consistentemente menor al obtenido por método convencional, podría ser un indicio de que este último no es totalmente efectivo para extraer la humedad de las astillas. Se hace referencia a que es posible que en el corazón de la muestra quede atrapada una porción de agua de hidratación la cual sería muy difícil de extraer a través del proceso de transferencia de calor que ocurre en una estufa. Debido a que la radiación de microondas actúa directamente sobre la molécula de agua aumentando su movilidad y permitiendo el calentamiento volumétrico de la muestra, esa proporción de agua de hidratación residual sería mucho más fácil de extraer cuando se utiliza la radiación de microondas en lugar de una estufa (Du et al., 2005; Bélanger et al., 2008; Calvino et al., 2008). Este hecho requiere ser estudiado con mayor detalle en trabajos posteriores.

La diferencia entre los resultados obtenidos para las muestras R10 y R11, podrían simplemente ser debido a que la muestra R11 contenía más agua libre o a las características particulares de cada rola, dependiendo del árbol y la altura a la cual se cortaron. En todo caso, a través de la comparación de los resultados obtenidos por el método convencional y HMO-1 y 2, se infiere que los métodos HMO podrían conducir a la determinación del factor de sequedad con mayor exactitud, debido a que es más eficiente al extraer la humedad de las astillas y supera las limitaciones impuestas por las características particulares de cada muestra.

Es importante destacar, que el comportamiento del proceso de pérdida de humedad observado (Figura 1) coincide con lo reportado por otros investigadores, en cuanto que al principio del proceso la pérdida de agua es mayor, para luego entrar en una fase intermedia y por último llegar a un peso constante (Du et al., 2005; Wang et al., 2005; Andersson et al., 2006). Adicionalmente, coincidimos en la observación que la utilización del horno de microondas permite extraer humedad con menos energía y menor incremento de la temperatura.

\section{Ahorro de energía}

Una de las motivaciones para desarrollar nuevos métodos de análisis empleando fuentes de energía alternativas, es la posibilidad de incrementar la eficiencia de los procesos y disminuir el consumo de energía, para coincidir 
con los principios de la química verde (Meléndes y Camacho, 2008; Calvino et al. 2008). La metodología descrita en este trabajo permite determinar el factor de sequedad de las astillas que serán utilizadas para la fabricación de pulpa para papel con un ahorro de energía del $99 \%$ y una reducción del tiempo de análisis de $90 \%$. Adicionalmente, los métodos HMO mostraron ser mejores en cuanto a su capacidad de proporcionar resultados más confiables, lo cual tendrá influencia directa en la determinación de las cargas que serán utilizadas en la fabricación de pulpa para papel, permitiendo optimizar el proceso y disminuir los costos de producción.

\section{CONCLUSIONES}

En este trabajo se desarrolló un método para determinar el contenido de humedad de astillas de Pinus caribaea var. hondurensis que serán utilizadas en la fabricación de pulpa para papel, la principal innovación consiste en emplear una fuente de energía térmica alternativa como es la radiación de microondas. Una de las características que se debe resaltar del nuevo método es que su implementación no requiere de grandes inversiones de dinero ni de la adquisición de equipos sofisticados, sólo es necesario adquirir un horno de microondas doméstico. Como lo demuestran los resultados esta metodología es eficiente y confiable, además de consumir un $99 \%$ menos de energía que el método convencional y reducir el tiempo análisis en un $90 \%$. Es importante resaltar que la calidad de este tipo de análisis incide directamente en la eficiencia y costos del proceso de pulpificación. Aunque, el procedimiento fue desarrollado para Pinus caribaea var. hondurensis puede tomarse como punto de partida para desarrollar métodos con otras especies.

Finalmente, se debe decir que es necesario incentivar el desarrollo de métodos de análisis dentro del concepto de la Química e Ingeniería Verde, ya que esto contribuirá al desarrollo de una industria papelera más amigable al ambiente.

\section{AGRADECIMIENTOS}

Este trabajo fue parcialmente financiado por la Empresa de Producción Social de Pulpa y Papel
C.A. (EPS-PULPACA) a través del Contrato de Servicios de UAPIT - ULA Nro. EPS- 009/2010, agradecemos especialmente la colaboración del Prof. Antonio Aguilera quien se desempeña actualmente como Gerente de Proyectos de la empresa y del Ing. MSc. Daniel Calderón por su apoyo en la realización del presente trabajo.

\section{REFERENCIAS BIBLIOGRÁFICAS}

Acierno, D., Barba, A. \& d'Amore, M. (2004). Heat transfer phenomena during processing materials with microwave energy. Heat and Mass Transfer, 40(5), 413-420.

Amazon.com. (2011). Amazon.com: Online Shopping. Recuperado de http://www.amazon . $\mathrm{com} /$ [Consultado el 25 octubre, 2011]

American Chemical Society.( 2001). ACS Green Chemistry Institute. Recuperado de http://portal. acs.org/portal/acs/corg/content?_nfpb=true\&_ pageLabel $=$ PP_TRANSITIONMAIN\&node_ id $=830 \&$ use_sec $=$ false \&sec_url

var=region $1 \&$ _ uuid=e 5 bf $345 \mathrm{e}-386 \mathrm{f}-442 \mathrm{e}-9575$ b6837928ed95[Consultado el 20 de agosto, 2011]

Andersson, M., Persson, L., Sjoholm, M. \& Svanberg, S. (2006). Spectroscopic studies of wood-drying processes. Optics Express, 14(8), 3641-3653.

Area, M. (2005). Tecnologías limpias para la producción de pulpa y papel. (pp. V-1 a V22). Concordia - Argentina: XX Jornadas Forestales de Entre Ríos.

ASTM D-4442-92.( 2003). Standart Test Methods for Direct Moisture Content Measurement of wood and wodd-based materials. Phil., $\mathrm{Pa}$, USA: ASTM Int.

Ayappa, K., Davis, H., Davis, E. \& Gordon, J.(1991). Analysis of microwave heating of materials with temperature-dependent properties. AICHE Journal, 37(3), 313-322.

Battana, B., Sharma, J., Sudha, S. \& Chander, R. (2007). Enhanced production of cellulasefree thermostable xylanase by Bacillus pumilus ASH and its potential application in paper industry. Enzyme and Microbial Technology, 41(6-7), 733-739

Bélanger, J., Paré, J., Poon, O., Fairbridge, C., Ng, S. \& Mutyala, S. (2008). Remarks on various 
applications of microwave energy. Journal of Microwave Power \& Electromagnetic Energy, 42(4), 24-44.

Breccia, A., Fini, A., González, M. \& González, M. (2003). Las microondas en los procesos químicos: efectos en la síntesis química y en los análisis químicos. Ingeniería Química, 407, 139-143.

Calvino, V., Martín, R. \& López, A. (2008). Catálisis heterogénea y radiación microondas, una tecnología alternativa para un desarrollo sostenible.Revista 100cias@uned, 116-121.

Cerón, L. (2009). Biotecnología en la industria de la pulpa, y el papel: mapeo de patentes. Revista EAN, 67, 139-154.

Cole-Parmer. (2011). Cole-Parmer Alphabetical Catalog. Recuperado de http://www. coleparmer.com/Catalog/\#O [Consultado el 25 octubre, 2011]

Du, G., Wang, S. \& Cai, Z. (2005). Microwave drying of wood strands. Drying Technolody, 23, 1-16.

Ferluc, A. (2008). Mirada hacia la industria papelera y su imagen medioambiental popular. Nuevo Mundo Mundos Nuevos, Cuestiones del tiempo presente. Recuperado de http://nuevomundo. revues.org/16142.html [Consultado el 20 agosto, 2011]

Gaete, H., Bay-Schmith, E. \& Riveros, A. (2005). Toxicidad crónica de las aguas receptoras de efluentes de industrias de celulosa sobre larvas de Chironomus piger. Revista Internacional de Contaminación Ambiental, 21(2), 83-89.

Hansson, L. \& Antti, A. (2006). The effect of drying method and temperature level on the hardness of wood. Journal of Materials Processing Technology, 171(3), 467-470.

Hunt, J., Gu, H., Walsh, P. \& Winandy, J. (2005). Developmente of new microwave-drying and straightening technology for low-value curved timber. National Fire Plan Research Program. USDA Forest Service, Forest Products Laboratory, Research Note FPL-RN-0296.

James, W., Yen, Y. \& King, R. (1985). A microwave method for measuring moisture content, density, and grain angle of good. USD Forest Service. USD Forest Service, Forest Product Laboratory, Research note FPL-0250.

Kappe, C. \& Dallinger, D. (2009). Controlled microwave heating in modern organic synthesis: highlights from the 2004-2008 literature. Molecular Drivers, 13(2), 71-193.

Meléndes, C. \& Camacho, A. (2008). Química verde: la química del nuevo milenio. Aventuras del pensamiento, 1-5.

Rowell, R. (2007). Chemical Modification of Wood. In S. Fakirov, D. Bhattacharryya, \& S. Fakirov (Ed.), Handbook of Enginiering Biopolymers: Homopolymers, blends and Composites (pp. 673-691). Munich, Germany: Hanser Publishers.

Schmidt, O. (2006). Wood and tree fungi: Biology, damage, protection, and use. (D. Czeschlik, Ed.) Berlin, Germany: Springer.

Soto, M. (2005). El consumo de papel y sus implicaciones sobre los bosques y el medioambiente. Cuadernos de Biodiversidad, 17, 21-26.

TAPPI. (1998). 1998-1999 TAPPI Test Methods. Atlanta - USA: TAPPI Press.

Treu, A., Rieche, H. \&Militz H. (2008). Spruce and pine heartwood treatment by means of microwave radiation. The International Research Group on Wood Protection, 39th Annual Meeting (pp. 1-10). Istanbul, Turkey: IRG Secretariat.

Wang, S., Du, G. \& Zhang, Y. (2005). Microwave Wood Strand Drying: Energy Consumption, VOC Emission and Dying Quality. IADC 2005 - 3rd Inter-American Drying Conference (pp. III-4). USA: IADC 2005 - 3rd Inter-American Drying Conference.

\section{SOBRE LOS AUTORES}

\section{Ilvania Gutiérrez Gotera}

Ingeniera Forestal de ULA.

Magister Scientiae en tecnología de Productos Forestales.

Personal de Investigación de la sección de Celulosa y Papel.

Correo electrónico: ilgutie@ula.ve

\section{Pedro R. Benítez Díaz}

Licenciado en Química. Magister Scientiae Ciencias Médicas Fundamentales. Profesor en la Universidad de Los Andes, Mérida,Venezuela en el Departamento de Botánica y Ciencias Básicas.

Correo electrónico: prbe@ula.ve 
